\title{
EXPERIENCIA DE EVALUACIÓN FORMATIVA EN EDUCACIÓN FÍSICA EN UN CENTRO RURAL EN SECUNDARIA
}

Experience of formative assessment in Physical Education in a rural center in secondary

Experiência de avaliação formativa na Educação Física num centro rural no ensino secundário

Teresa Fuentes Nieto (1)

(1) IES Marqués de Lozoya, Cuellar (Segovia), España. Teléfono: +34609426549. Correo electrónico: fuentes.teresa@gmail.com

\section{Resumen}

La experiencia de evaluación formativa y compartida se lleva a cabo en el IES Marqués de Lozoya en Cuellar (Segovia), durante el curso 2018/2019, en 2 grupos de $3^{\circ}$ de ESO con un total de 38 alumnos en la asignatura de Educación Física (2 horas semanales). A lo largo del curso se lleva a cabo una evaluación formativa y compartida que se caracteriza por el uso de las técnicas de evaluación de observación y reflexión; así como la utilización rúbricas de autoevaluación, coevaluación y heteroevaluación y fichas de observación como principales instrumentos de evaluación. Los resultados obtenidos en las diferentes evaluaciones han sido positivos y han mejorado de la $1^{\circ}$ a la $2^{\circ}$ evaluación. Podemos concluir que la utilización de este tipo de evaluación aumenta la implicación por parte del alumnado y del profesor mejorando así su aprendizaje y resultado académico.

Palabras clave: Evaluación formativa y compartida, secundaria, educación física

\begin{abstract}
The experience of formative and shared assessment is carried out in the Marqués de Lozoya High School of Cuellar (Segovia), during the 2018/2019 academic year, in the 2 groups of $3^{\circ}$ of ESO with a total of 38 students in the subject of Physical Education (2 hours per week). Throughout the course, a formative and shared evaluation is carried out, which is characterized by the use of observation and reflection assessment techniques; as well as the use of self-assessment, peer-assessment and hetero-evaluation
\end{abstract}


rubrics and observation sheets as the main evaluation instruments. The results obtained in the different evaluations have been positive and have improved from the 1 st to the 2nd trimester. We can conclude that the use of this type of assessment increases the involvement of the students and the teacher, thus improving their learning and academic results.

Keywords: Formative and shared assessment, secondary, physical education

\section{Resumo}

A experiência de avaliação formativa e partilhada foi realizada no IES Marques de Lozoya em Cuellar (Segóvia), durante o ano letivo 2018/2019, em dois grupos de 3. ${ }^{\circ}$ da ESO, que conta com um total de 38 alunos na disciplina de Educação Física (2 horas por semana). Ao longo do curso foi realizada uma avaliação formativa e partilhada, caracterizada pelo uso de técnicas de avaliação de observação e reflexão; assim como o uso de rubricas de autoavaliação, coavaliação e heteroavaliação e fichas de observação como principais instrumentos de avaliação. Os resultados obtidos nas diferentes avaliações foram positivos e melhoraram da $1 .^{\mathrm{a}}$ para a 2. ${ }^{\mathrm{a}}$ avaliação. Podemos concluir que a utilização deste tipo de avaliação aumenta o envolvimento dos alunos e do professor, melhorando assim a sua aprendizagem e resultados académicos.

Palavras-chave: Avaliação formativa e partilhada, secundária, educação física

\section{Introducción}

En este trabajo se expone la aplicación de la evaluación formativa y compartida (LópezPastor, 2006; López-Pastor et al., 2013) en la asignatura de Educación Física en la etapa de Educación Secundaria durante el curso escolar 2018/2019. Se describen los principales los sistemas de evaluación y calificación utilizados, incluyendo ejemplos de los instrumentos de evaluación aplicados. Por último, se exponen y discuten los resultados obtenidos.

\section{Contextualización}

La experiencia se lleva a cabo en el IES Marqués de Lozoya de Cuellar (Segovia), durante el curso 2018/2019. Es un centro de unos 500 alumnos con una media de 3 grupos por curso de ESO y bachillerato, con sección bilingüe. 
El informe se centra la asignatura de Educación Física de $3^{\circ}$ de ESO, con una carga lectiva de 2 horas semanales, impartida por la misma profesora en 2 grupos $3^{\circ} \mathrm{A}-\mathrm{PMAR}$ (20 alumnos) y $3^{\circ} \mathrm{C}$ (18 alumnos).

\section{Diseño de la propuesta formativa}

a) Procedimientos de evaluación formativa

La técnica predominante de evaluación formativa es la observación y reflexión. El feeback se da entre iguales y del profesor hacia los alumnos. Se trata de un feedback inmediato o concurrente (simultáneo a la acción); suele ser individual o en pequeños grupos (si es útil en grupo entero). Solo en el caso de los días de presentación de una producción final el feedback es menos inmediato.

Los alumnos son informados de cómo se les va a evaluar y al inicio de cada Unidad Didáctica (en adelante UD) (rúbricas, criterios de evaluación concretos...).

b) Sistema de calificación

A pesar de que la evaluación es continua, se debe dar una calificación en el $1^{\circ}$ y $2^{\circ}$ trimestre además de la final (tercer trimestre). Se da un peso concreto a cada UD dentro del trimestre. De forma genérica, la actitud supone un 30\% de la calificación y el resto corresponde a aspectos procedimentales (40\%) y conceptuales $(30 \%)$. La nota final tiene en cuenta las actividades de todo el curso. Los porcentajes en la calificación de las principales actividades de aprendizaje se exponen en la figura resumen de actividades aprendizaje (Figura 1, en Anexos). Asimismo, en las Figuras 2, 3 y 4 (en Anexos) y se muestran a modo de ejemplo algunos de los instrumentos formativos empleados.

Además, se utiliza el cuaderno del profesor digital "additio" (https://www.additioapp.com/es).

El profesor dispone también de un cuaderno abierto, no sistemático para la anotación de aspectos relacionados con la planificación de la asignatura, anécdotas, ideas y reflexiones.

\section{Efectos de la implementación de la propuesta evaluadora formativa}

Resultados-efecto en el rendimiento académico del alumnado:

Los resultados se referirán a la $1^{\mathrm{o}}$ y $2^{\circ}$ evaluación, ya finalizadas y calificadas.

Los alumnos cada vez se ajustan mejor a los criterios de evaluación, con una coevaluación y autoevaluación muy similar a la heteroevaluación del profesor. En la 
segunda evaluación no hay ningún alumno suspenso, hay menos "aprobados", más del doble de calificaciones de "bien" y hay 2 alumnos más que alcanzan el sobresaliente (Tabla 1).

Tabla 1.

Resultados académicos

\begin{tabular}{ccccc}
\hline \multicolumn{4}{c}{ Resultados globales } \\
\hline Calificación & \multicolumn{2}{c}{$\mathbf{1}^{\mathbf{0}}$ Evaluación } & \multicolumn{2}{c}{$\mathbf{2}^{\mathbf{0}}$ Evaluación } \\
Sobresaliente & $\mathbf{P o r c e n t a j e}$ & $\mathbf{N}^{\mathbf{0}}$ alumnos/as & Porcentaje & $\mathbf{N}^{\mathbf{0}}$ alumnos/as \\
Notable & $50 \%$ & 1 & $7.9 \%$ & 3 \\
Bien & $10.5 \%$ & 19 & $44.7 \%$ & 17 \\
Aprobado & $31.5 \%$ & 4 & $26.3 \%$ & 10 \\
Suspenso & $5.3 \%$ & $21.1 \%$ & 8 \\
No presentado & $0 \%$ & 0 & $0 \%$ & 0 \\
Totales & $\mathbf{1 0 0 \%}$ & $\mathbf{3 8}$ & $0 \%$ & 0 \\
\hline
\end{tabular}

Todos los alumnos han alcanzados los mínimos requeridos para superar la asignatura en la $2^{\mathrm{a}}$ evaluación y más de un $50 \%$ de los alumnos obtienen una calificación por encima del 7 en ambas evaluaciones, un éxito teniendo en cuenta el número de alumnos y que 10 de ellos pertenecen al Programa de Mejora del Aprendizaje y el Rendimiento (PMAR).

La poca existencia de sobresalientes puede deberse a la adaptación paulatina por parte de los alumnos a la evaluación formativa. Esto puede explicar los 2 alumnos más con sobresaliente en la $2^{a}$ evaluación y el aumento considerable de "bienes" y reducción de “aprobados". Destaca la ausencia de suspensos en la $2^{\mathrm{a}}$ evaluación, en este sentido, la profesora explicó a los 2 alumnos suspensos, ambos pertenecientes al PMAR, la dinámica de evaluación y cómo poder superar la asignatura.

\section{Conclusiones}

La experiencia plasmada muestra cómo la profesora está adaptando su asignatura a la evaluación formativa y cómo está obteniendo buenos resultados. Algunas de las actividades de aprendizaje llevadas a cabo como la elaboración de los tutoriales de bachata, reflejan el potencial de los alumnos a la hora de construir su propio aprendizaje y las virtudes de la evaluación formativa. Las ventajas principales son:

- Coherencia entre los objetivos, las actividades de aprendizaje y la evaluación.

- Alumno responsable de su aprendizaje.

- Mejor diálogo entre alumnos y entre el docente y los alumnos.

- Mejor ajuste de los objetivos y los resultados. 
-Mayor reflexión sobre las actividades de aprendizaje, ajuste y mejora en la planificación presente y futura por parte del profesor.

\section{Referencias}

López-Pastor, V. M., Kirk, D., Lorente-Catalán, E., MacPhail, A., \& Macdonald, D. (2013). Alternative Assessment in Physical Education: A Review of International Literature. Sport, Education \& Society, 18(1), 57-76. DOI:10.1080/13573322.2012.713860.

López-Pastor, V. M. (Coord.) (2006). La Evaluación en Educación Física: revisión de los modelos tradicionales y planteamiento de una alternativa: la Evaluación Formativa y Compartida. Buenos Aires: Miño y Dávila. 


\begin{tabular}{|c|c|c|c|c|}
\hline $\begin{array}{l}\text { Finalidades y/o } \\
\text { Corpetencias }\end{array}$ & $\begin{array}{l}\text { Actividades } \\
\text { Aprendizaje }\end{array}$ & Evaluacion formativa & $\begin{array}{l}\text { Instrumentos } \\
\text { calificacion }\end{array}$ & $\begin{array}{l}\text { Criterios } \\
\text { calificacion }\end{array}$ \\
\hline \multirow{5}{*}{$\begin{array}{l}\text { Valorar la actividad } \\
\text { fisica y la dieta } \\
\text { equilibrada como } \\
\text { habitos saludables. }\end{array}$} & $\begin{array}{l}\text { Test fisicos } \\
\text { relacionados conla } \\
\text { salud. }\end{array}$ & $\begin{array}{l}\text { Autonomia enla } \\
\text { realización y valoracion } \\
\text { de los test. }\end{array}$ & Ficha de test. & $\begin{array}{l}10 \% \\
\left(1^{\circ} \text { y } 3^{x}\right. \\
\text { evaluacion) }\end{array}$ \\
\hline & $\begin{array}{l}\text { Juegos para } \\
\text { categorizar alimentos. }\end{array}$ & $\begin{array}{l}\text { Reflexion tas los juegos } \\
\text { de los tipos de alinentos } \\
\text { (macro nutientes y } \\
\text { saludables). }\end{array}$ & $\begin{array}{l}\text { Observacion y } \\
\text { anedotaio. }\end{array}$ & $\begin{array}{l}\text { Sin peso } \\
\text { (transversal) } \\
2^{\circ} \text { y } 3^{\circ} \\
\text { evaluacion }\end{array}$ \\
\hline & $\begin{array}{l}\text { Metodos variados } \\
\text { para el trabajo de la } \\
\text { condicion fisica (CF). } \\
\text { juegos y "correra } \\
\text { ritumo". }\end{array}$ & $\begin{array}{l}\text { Responder las pregmitas } \\
\text { sobre el entrenamiento, } \\
\text { cuantificacion y mejora } \\
\text { de la CF. }\end{array}$ & $\begin{array}{l}\text { Ficha de correr a } \\
\text { ritmo. }\end{array}$ & $\begin{array}{l}20 \% \\
\left(1^{\circ} \text { evaluacion }\right)\end{array}$ \\
\hline & $\begin{array}{l}\text { Registro dela dieta } \\
\text { semanal. }\end{array}$ & $\begin{array}{l}\text { Registro senranal: } \\
\text { conocimiento y reflaxion } \\
\text { de su dieta habitual. }\end{array}$ & $\begin{array}{l}\text { Ficha de registro de } \\
7 \text { dias. }\end{array}$ & $\begin{array}{l}10 \% \\
\left(1^{\circ} \text { evaluacion) }\right.\end{array}$ \\
\hline & Diseño dieta sentanal & $\begin{array}{l}\text { Revision de memis } \\
\text { saludables. Feadback }\end{array}$ & $\begin{array}{l}\text { Ficha de meml } \\
\text { semanal. }\end{array}$ & $\begin{array}{l}10 \% \\
\left(3^{\circ} \text { evaluacion) }\right.\end{array}$ \\
\hline \multirow[t]{2}{*}{$\begin{array}{l}\text { Realizar trabajo } \\
\text { cooperativo }\end{array}$} & $\begin{array}{l}\text { Corrbas cooperaivas. } \\
\text { Retos cooperativos. }\end{array}$ & $\begin{array}{l}\text { Evaluacion de montaje } \\
\text { final grupal }\end{array}$ & $\begin{array}{l}\text { Rubrica de } \\
\text { autoevahuacion, } \\
\text { coevaluadiony. } \\
\text { heteroevaluacion. }\end{array}$ & $\begin{array}{l}30 \% \\
\left(1^{\circ} \text { evaluacion) }\right.\end{array}$ \\
\hline & $\begin{array}{l}\text { Deportes altemaivos: } \\
\text { ringol y pinfurvote. } \\
\text { Tomeos. }\end{array}$ & $\begin{array}{l}\text { Evaluacion de roles y } \\
\text { registro en situacion de } \\
\text { juego. }\end{array}$ & $\begin{array}{l}\text { Rubricas de } \\
\text { autoevahucion, } \\
\text { coevaluaciony. } \\
\text { heteroevaluacion. }\end{array}$ & $\begin{array}{l}20 \% \\
\left(3^{\circ} \text { evaluxion) }\right.\end{array}$ \\
\hline \multirow[t]{2}{*}{$\begin{array}{l}\text { Participar } \\
\text { activamente en } \\
\text { actividades en el } \\
\text { madio natual }\end{array}$} & $\begin{array}{l}\text { Juegos y pruebas de } \\
\text { orientacion. } \\
\text { Resolucion de } \\
\text { pruebas de } \\
\text { orientacion por } \\
\text { grupos. }\end{array}$ & $\begin{array}{l}\text { Registro derealizadion } \\
\text { delas pruebas de } \\
\text { orientacion. }\end{array}$ & $\begin{array}{l}\text { Fichas de } \\
\text { orientacion: } \\
\text { autoevahacion, } \\
\text { heteroevaluacion y } \\
\text { coevaluacion. }\end{array}$ & $\begin{array}{l}20 \% \\
\left(3^{\circ} \text { evaluacion) }\right.\end{array}$ \\
\hline & $\begin{array}{l}\text { Ciclismo: rutas y } \\
\text { mantenimiento y } \\
\text { cuidado de la } \\
\text { bicicleta. }\end{array}$ & $\begin{array}{l}\text { Finalizacion de las rutas } \\
\text { y manejo de la bicidas y } \\
\text { de su mantenimiento. }\end{array}$ & $\begin{array}{l}\text { Fichas de } \\
\text { observacion (ain } \\
\text { sin deteminar) }\end{array}$ & $\begin{array}{l}20 \% \\
\left(3^{\circ} \text { evaluacion }\right)\end{array}$ \\
\hline $\begin{array}{l}\text { Realizar actividades } \\
\text { expresivas con } \\
\text { soporterusical }\end{array}$ & $\begin{array}{l}\text { Bachata: aprendizaje } \\
\text { de pasos y ruada de } \\
\text { bachata. } \\
\text { Elaboracion de un } \\
\text { tutorial por grupos. }\end{array}$ & $\begin{array}{l}\text { Autoevaluacion, } \\
\text { coevaluaciony } \\
\text { heteroevaluacion de los } \\
\text { tutoriales a taves de } \\
\text { plickers } \\
\text { (https:/getplickers com } \\
\text { ). }\end{array}$ & $\begin{array}{l}\text { Cuestionario de } \\
\text { preguntas concretas } \\
\text { sobre el turorial a } \\
\text { traves de pliclers } \\
\text { (autoevahucion. } \\
\text { coevaluaciony. } \\
\text { heteroevaluacion). }\end{array}$ & $\begin{array}{l}20 \% \\
\left(2^{2} \text { evaluacion) }\right.\end{array}$ \\
\hline $\begin{array}{l}\text { Participadion en } \\
\text { tomeos de diferentex } \\
\text { deportes }\end{array}$ & $\begin{array}{l}\text { Actividades de } \\
\text { aprendizaje tacnico- } \\
\text { tactico de Rugby tag } \\
\text { y badiminton. Tomeos } \\
\text { individuales y por } \\
\text { parejas y porequipos. }\end{array}$ & $\begin{array}{l}\text { Observacion en situacion } \\
\text { de juego y resultados en } \\
\text { los tomaos. }\end{array}$ & $\begin{array}{l}\text { Ficha de } \\
\text { observacion no } \\
\text { sistematicas para } \\
\text { evaluar el rolde } \\
\text { jugador y de } \\
\text { arbitro. }\end{array}$ & $\begin{array}{l}40 \% \\
\left(2^{\circ} \text { evaluacion) }\right.\end{array}$ \\
\hline
\end{tabular}

Figura 1. Coherencia interna entre los elementos curriculares. Alineación curricular. 


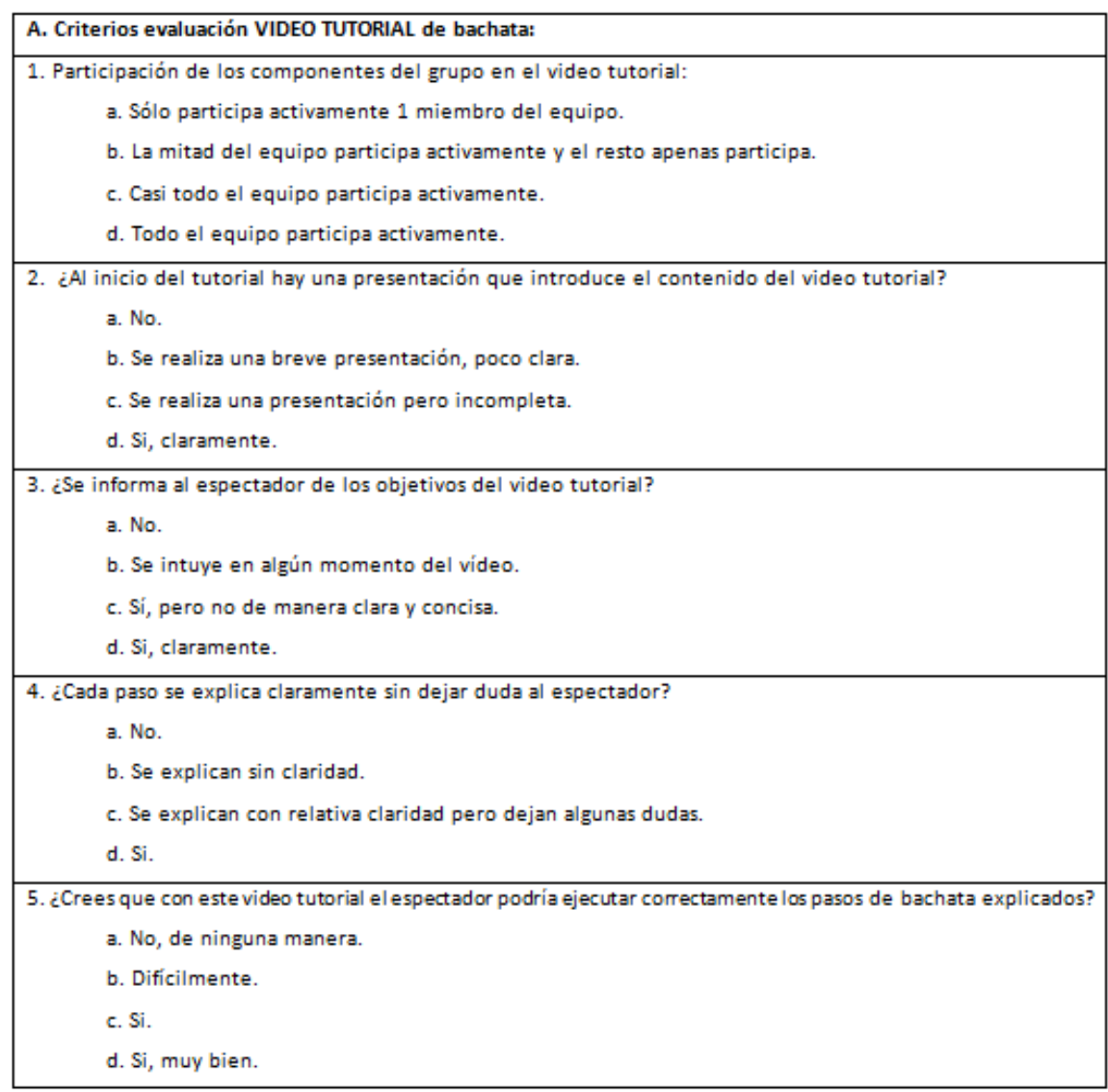

Figura 2. Escala descriptiva para la evaluación del video-tutorial de bachata.

CLASE: Grupo evaluador (nombre y componentes):

\begin{tabular}{|c|c|c|c|c|}
\hline $\begin{array}{l}\text { Rellenad entre todos esta } \\
\text { ficha una vez terminada la } \\
\text { actuación del grupo a } \\
\text { evaluar. }\end{array}$ & CRITERIOS DE EVALUCIÓN & $\begin{array}{l}\text { Giupo I (nombre y } \\
\text { compeosentes): }\end{array}$ & $\begin{array}{l}\text { Grupo } 2 \text { (nombre y } \\
\text { componentss): }\end{array}$ & $\begin{array}{l}\text { Grupo } 3 \text { (nomblore y } \\
\text { componemes): }\end{array}$ \\
\hline Organización. & $\begin{array}{l}\text { 4- Todos los componentes del grupo saben lo que tienen que hacer en todo } \\
\text { momento. } \\
\text { 2- En más de dos ocasiones se aprecia desorientación de uno o más compañeros, } \\
\text { sin saber que tiene que hacer. } \\
\text { 0- En prácticamente toda la composición casi nadie sabe lo que hay que hacer y } \\
\text { que viene después. }\end{array}$ & & & \\
\hline Participación. & $\begin{array}{l}3 \text { - Todos los componentes del grupo participan activamente en la mayoria de las } \\
\text { actividades de la composición. } \\
\text { 1- Hay al menos un compañero que no participa, o hasta } 3 \text { compañeros que } \\
\text { participan poco en comparación con los demás. } \\
\text { 0- Hay hasta } 3 \text { compañeros que no participan prácticamente nada. }\end{array}$ & & & \\
\hline Ejecución. & $\begin{array}{l}\text { 3- Todos los elementos y actividades seleccionadas se realizan correctamente y } \\
\text { se adecuan a la música. } \\
\text { 1- Se falla en más de } 2 \text { ocasiones y la ejecución no se adecua apenas a la música. } \\
\text { 0- Se falla reiteradamente. Más de la mitad de las veces. La música no tiene nada } \\
\text { que ver. }\end{array}$ & & & \\
\hline Variedad & $\begin{array}{l}\text { 3- Se realizan saltos diferentes, entradas a la comba desde diversos lados, } \\
\text { combinaciones con los compañeros distintas... } \\
\text { 1- Casi siempre se entra desde el mismo lado, con saltos simples, muy poca } \\
\text { combinación en las formas de interaccionar con los compañeros y poca variedad. }\end{array}$ & & & \\
\hline Creatividad. & $\begin{array}{l}4 \text { - Varias de las actividades y saltos seleccionados son originales y generan } \\
\text { sorpresa al ser vistos. } \\
2 \text { - Al menos } 2 \text { elementos son creativos o diferentes. } \\
0 \text { - Todos son saltos y actividades ya realizadas y sin ningún tipo de } \\
\text { combinación entre ellas. }\end{array}$ & & & \\
\hline Composición global. & $\begin{array}{l}3 \text { - Lo realizado muestra que se ha puesto bastante interés y ganas por realizar } \\
\text { una buena composición. } \\
1 \text { - El interés ha sido poco. Se aprecia poco trabajo. }\end{array}$ & & & \\
\hline TOTAL & - Suma de todas las puntuaciones. & & & \\
\hline
\end{tabular}

Figura 3. Escala de puntuación de la composición de combas cooperativas. 


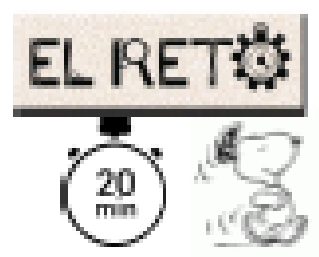

Fichas de registro

UD2: Condición Fícica. Resistencis. Correr a ritmo

\begin{tabular}{|l|l|}
\hline DíA 1. & FC reposo: $\quad \mathrm{Pm}$ \\
\hline Fecho: & FC post-carrera: \\
\hline
\end{tabular}

RETO: No parar durante 20 minutos. iilCarrer el mbximo tiempo posible!!!

INDICACIONES:

- Corresollo o acompañado (mejor con compañeros que creos que irdán a tu misma velocidad).

- Debes controlar el tiempe: $20^{\circ}$.

- Cuenta las vueltes que das al circuito.

NÚMERO DE WUELTAS ( $n^{\circ}$ vueltas):

TIEMPO MEDIO POR VUELTA (tiempo total $/ \mathrm{n}^{\circ}$ vuel tes):

Eje: 12 minutos/ 7 weltas $=1.71$ es decir $1 \mathrm{~min} 43^{\prime \prime}$ (para pasar a segundos: 71060/100)

CHAS TENIDO QUE PARAR DE CORRER? Si No

- LCudntas veces?

- ¿Durante cuánto tiempo?

- ¿Por qué crees que has tenido que caminar?

¿Qué es la resistencia? ¿Que sistemas de producción de energía tiene nuestro cuerpo? ¿Qué tipos de resistencia conoces?

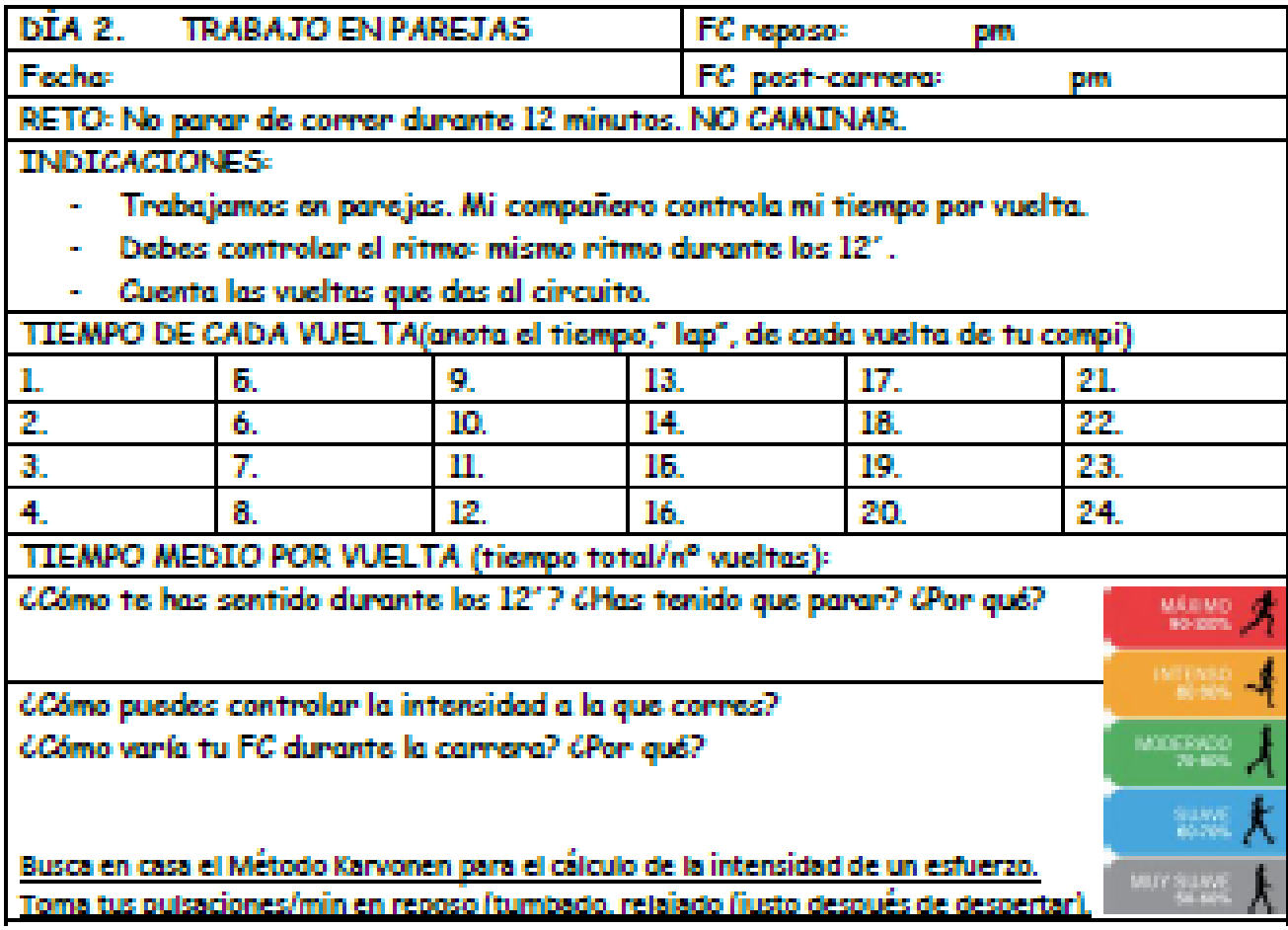

"Es obligatorio rellenar todos los huecos y contestar a todas las preguntas razonando. 


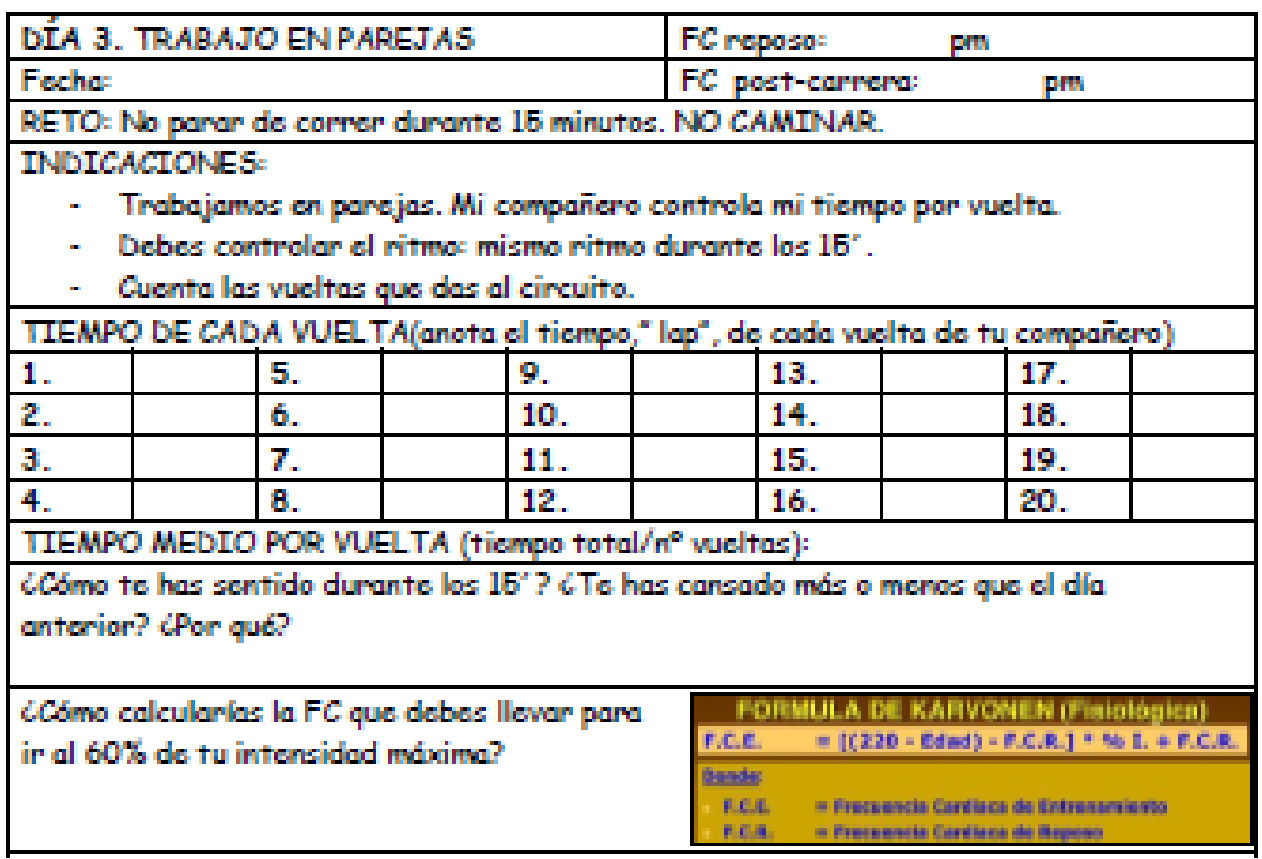

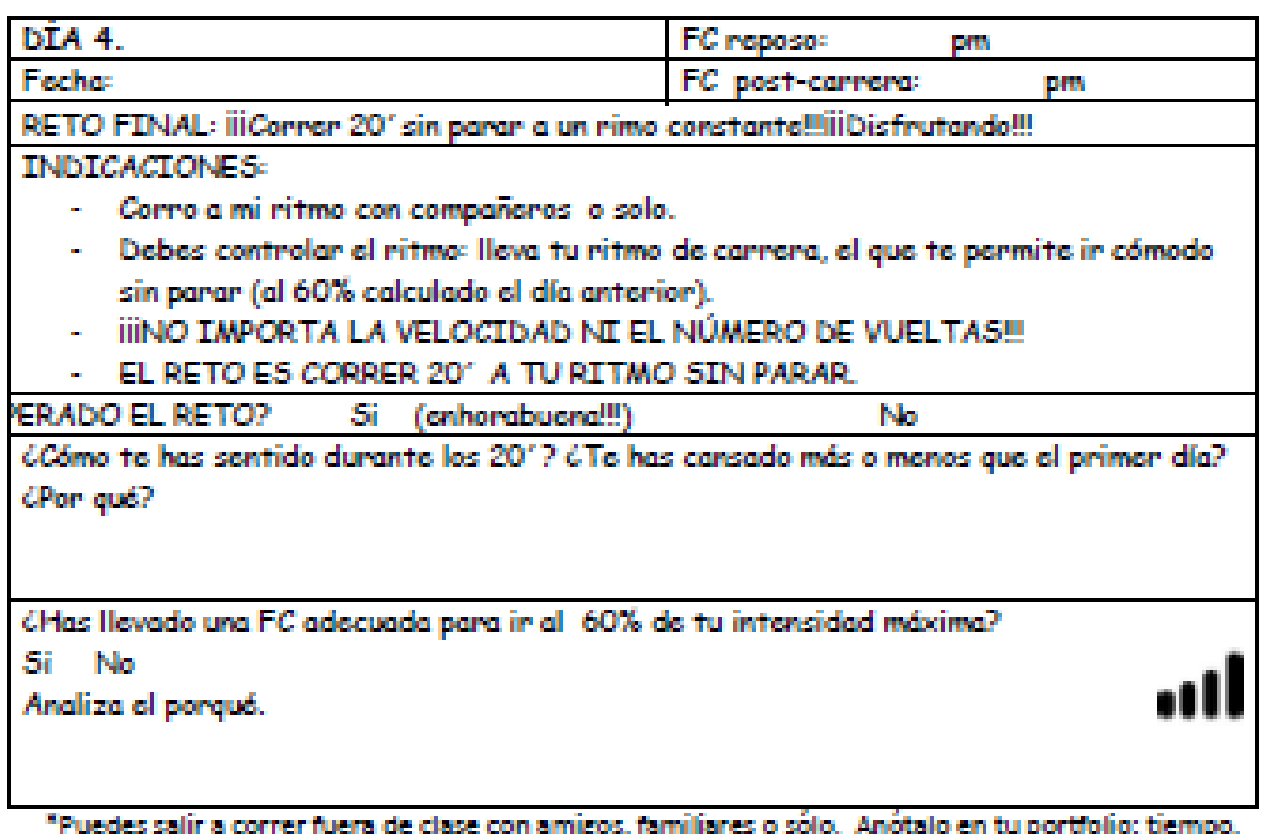

recorrido, con quëén corres.

"Es obligatorio rellenar todos los huecos y contestar a todss les preguntas razonando.

Figura 4. Ficha evaluación de "correr a ritmo" 